\title{
Yield Gap of Stress Tolerant Rice Varieties Binadhan-10 \& Binadhan-11 in Some Selected Areas of Bangladesh
}

\author{
Razia Sultana, Md. Habibur Rahman, Md. Rashidul Haque, Md. Mohsin Ali Sarkar, Syful Islam \\ Bangladesh Institute of Nuclear Agriculture (BINA), Mymensingh, Bangladesh \\ Email: razia1201@gmail.com
}

How to cite this paper: Sultana, R., Rahman, Md.H., Haque, Md.R., Sarkar, Md.M.A. and Islam, S. (2019) Yield Gap of Stress Tolerant Rice Varieties Binadhan-10 \& Binadhan-11 in Some Selected Areas of Bangladesh. Agricultural Sciences, 10, 1438-1452. https://doi.org/10.4236/as.2019.1011105

Received: October 6, 2019

Accepted: November 16, 2019

Published: November 19, 2019

Copyright () 2019 by author(s) and Scientific Research Publishing Inc. This work is licensed under the Creative Commons Attribution International License (CC BY 4.0).

http://creativecommons.org/licenses/by/4.0/

\section{(c) (i) Open Access}

\begin{abstract}
Binadhan-10 \& Binadhan-11 are climate smart stress tolerant high yielding rice varieties (yield $>4 \mathrm{t} \cdot \mathrm{ha}^{-1}$ ) have saline tolerant EC up to $12 \mathrm{ds} / \mathrm{m}$ and submergence tolerant up to 20 - 25 days capacity. The present study was an attempt to analyze the yield gap of stress tolerant varieties Binadhan-10 \& Binadhan-11 in some selected areas of Bangladesh. The objectives of the study were: 1) to estimate the yield gap of Binadhan-10 \&-11 growers among the study areas; 2 ) to identify the factors affecting the yield of these variety; and 3) to suggest some policy guidelines to minimize the yield gap. The study was conducted in eight Binadhan-10 \& Binadhan-11 growing areas in Bangladesh. In this study, four districts namely Satkhira, Khulna, Barishal, and Cox's Bazar were used for Binadhan-10 and Mymensingh, Jamalpur, Sherpur and Sunamgonj were taken for Binadhan-11. It is based on primary level data from eight sub-districts among the study areas. A total of 240 farmers were randomly selected (30 from each location) to collect the data with a pre-designed questionnaire. Farmer were grouped according to saline affected and not-affected for saline tolerant variety Binadhan-10 and not affected, affected ( 1 - 10 days) and highly affected (10 - 20 days) for submergence tolerant rice variety Binadhan-11 to identify existing yield gap. Tabular as well as Zandstra method were applied for analysis the data. The study also found factors affecting the gap and some policy guidelines to minimize the gap.
\end{abstract}

\section{Keywords}

Stress Tolerant, Saline Tolerant, Submergence Tolerant, Rice, Yield, Yield Gap 


\section{Introduction}

Global environment is changing due to change in temperature in alarming rate. Rice is the crop of the Asia-Pacific Region, but production of rice is hampering due to change in climate. In other hand yield gap causes lowering in production. The projected demand of rice by the year 2025 is mind boggling [1], as in major Asian countries rice consumption will increase faster than the population growth. Demand for rice is expected to grow faster than the production in most countries [2] so much so that by 2025 we will need 800 million tonnes of it annually. The population of Bangladesh will reach near about 215 million in 2050. So food grain must be increased for upcoming future generation. At that time requirement of rice will be 44.6 million metric tonnes. In summary, in Asia, the rice consumption by the year 2025, over the base year 1995, will increase by more than 51 percent (Table 1). The highest projected population was observed China i.e. 1471 million in 2025 and the lowest was observed in Rep. of Korea i.e. 52.9 million (Figure 1). But in case of percentage change, it was the highest in Pakis$\tan$ i.e. $87 \%$ and the lowest in Japan i.e. $-1 \%$ (Figure 2).

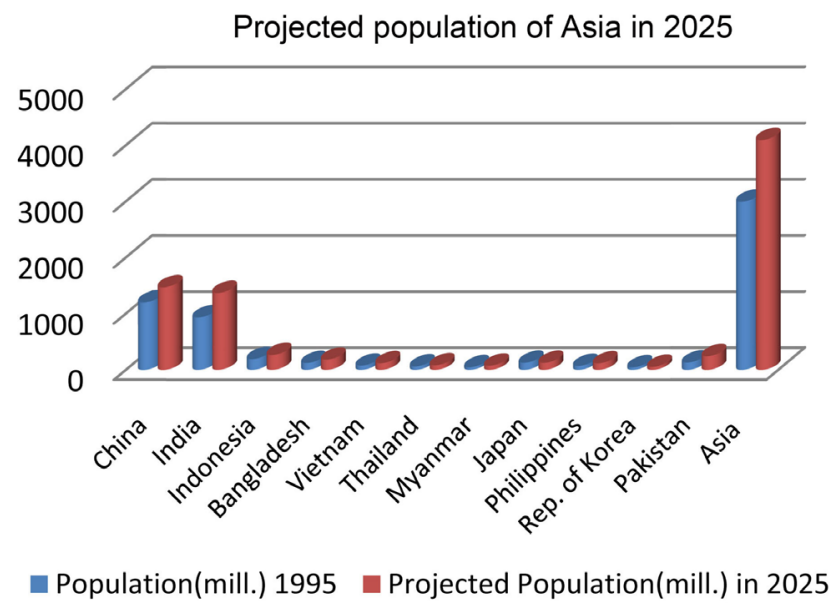

Figure 1. Bar diagram showing projected population in 2025 in Asia.

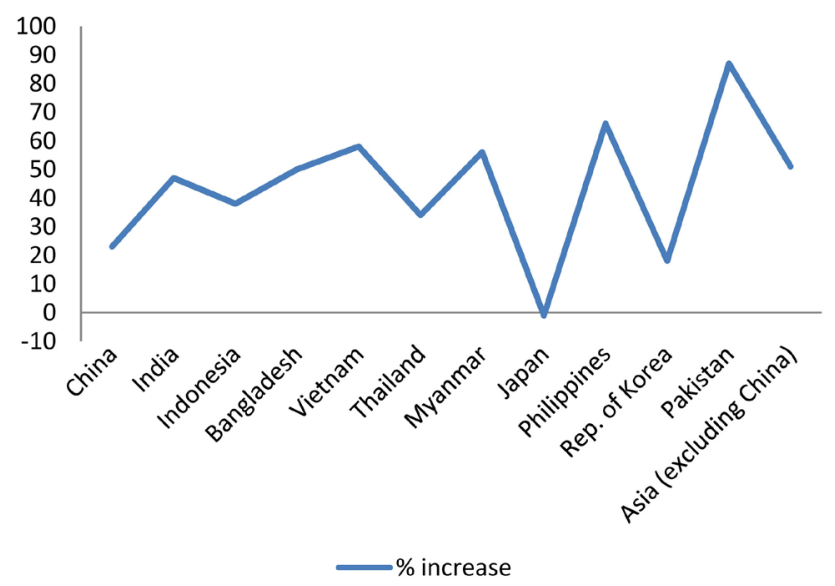

Figure 2. Percentage change of population in Asia. 
Table 1. Population growth rate and projected population in Asian countries.

\begin{tabular}{cccccc}
\hline Country & $\begin{array}{c}\text { Population } \\
\text { (mill.) } 1995\end{array}$ & $\begin{array}{c}\text { Annual Growth Rate } \\
\text { (\% per year) }\end{array}$ & $\begin{array}{c}\text { Projected } \\
\text { Population }\end{array}$ & $\begin{array}{c}\text { Percent } \\
\text { Increase }\end{array}$ \\
\cline { 3 - 4 } China & 1199 & 0.9 & 0.5 & 1471 & 23 \\
India & 934 & 1.7 & 1.0 & 1370 & 47 \\
Indonesia & 192 & 1.4 & 0.8 & 265 & 38 \\
Bangladesh & 121 & 1.8 & 1.1 & 182 & 50 \\
Vietnam & 74.1 & 2.0 & 1.2 & 117 & 58 \\
Thailand & 60.5 & 1.3 & 0.7 & 80.8 & 34 \\
Myanmar & 46.8 & 2.1 & 1.1 & 72.9 & 56 \\
Japan & 125 & 0.3 & -0.3 & 124 & -1 \\
Philippines & 69.2 & 2.2 & 1.2 & 115 & 66 \\
Rep. of Korea & 44.8 & 0.8 & 0.3 & 52.9 & 18 \\
Pakistan & 130 & 2.7 & 1.6 & 243 & 87 \\
Asia (excluding China) & 2244 & 1.8 & 1.1 & 3389 & 51 \\
\hline
\end{tabular}

Source :Word Bank population projection, 1994-95 [3].

Figure 3 shows the global map of current percentage rates of changes in different field crops yield such as: 1) maize, 2) rice, 3) wheat, and 4) soybean. In this figure, the red areas in the map showed where yields are decreasing whereas the fluorescent green areas show where rates of yield increase if continued it would twice over production by 2050 .

In Bangladesh, various high yielding stress tolerant rice varieties have been developed by different institute \& organization. Binahan-10 \& Binadhan-11 are the two stress tolerant varieties develop by BINA. Binadhan-10 is a salt tolerant variety for boro season which can tolerate up to $12 \mathrm{dS} / \mathrm{m}$ of salinity, released in 2012. The variety is capable to produce higher seed yield (5 - $6 \mathrm{t} / \mathrm{ha}$ under salt stress). Binadhan-10 is early maturing (127 - 132 days) than other salt tolerant varieties. In non-saline condition, potential yield is $8.5 \mathrm{t} / \mathrm{ha}$ (average $7.5 \mathrm{t} / \mathrm{ha}$ ). Grain is medium long, slender and bright colour. The variety is suitable for both aman and boro seasons. Binadhan-11 has been released as a submergence tolerant early maturing rice variety for aman season. This variety possesses green and erect flag leaves. Duration of this variety is 130 - 135 days (under 20 - 25 days submerged condition) and 115 - 120 days for non-submerged condition. In submerged condition, potential yield is $4.5 \mathrm{t} / \mathrm{ha}$ (average $4.2 \mathrm{t} / \mathrm{ha}$ ). This variety is capable to produce 5.0 - $5.4 \mathrm{t} / \mathrm{ha}$ in non-submerged condition. Despite the technologies developed by different National Agricultural Research System (NARS) institutes and extension agencies to disseminate the technologies, yield gaps exist in different crops of Bangladesh, such as rice, wheat, potato, oilseeds, pulses, etc. that may range from $19 \%$ to about $64 \%$ of the potential yield [5]-[11]. The existence of yield gaps was also observed in rice, mustard, and cotton in India 


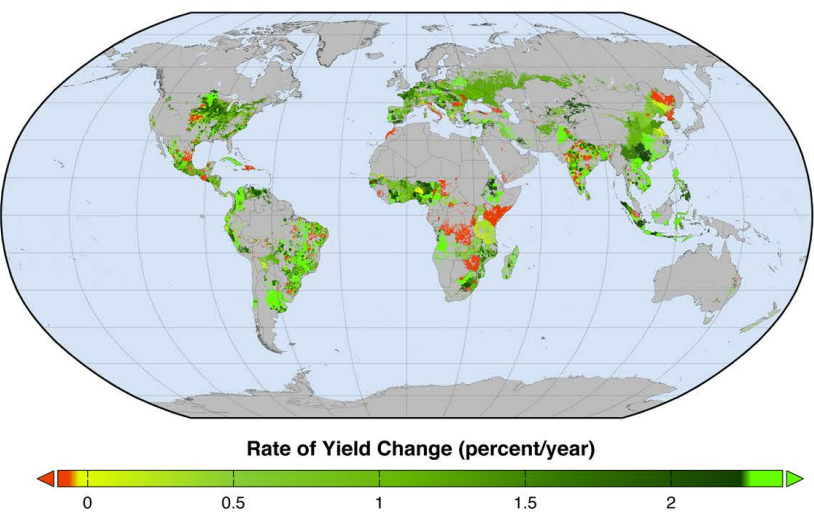

(a)

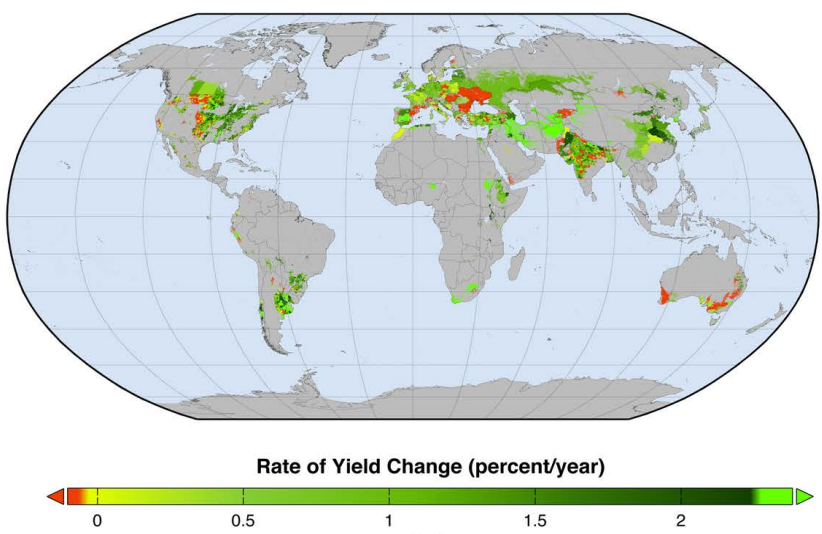

(c)

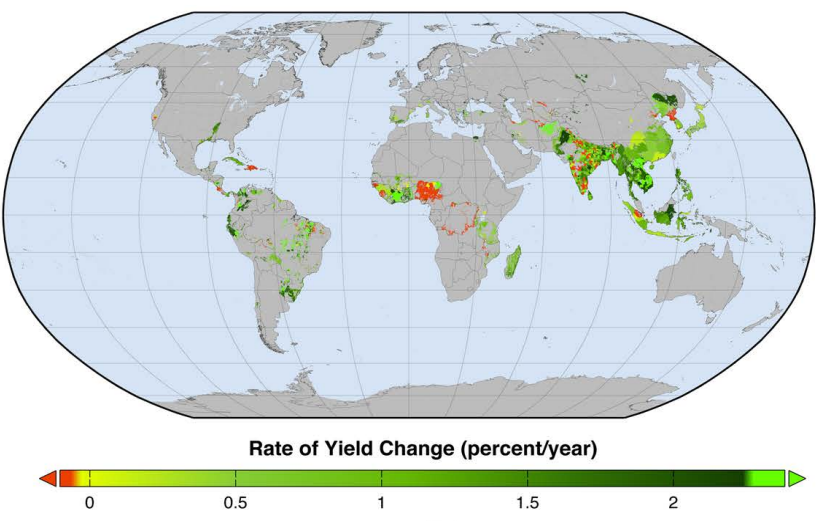

(b)

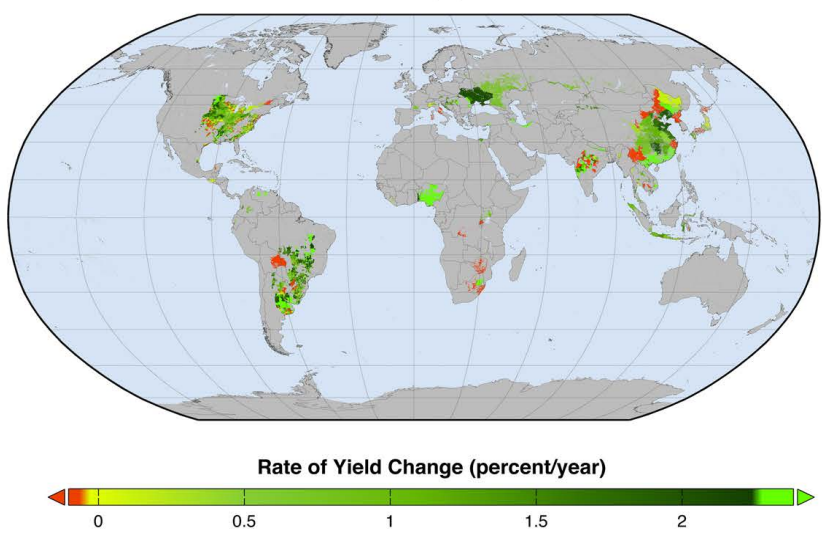

(d)

Figure 3. Maps of observed rates of percent yield changes per year [4]. (a) Maize; (b) Rice; (c) Wheat; (d) Soybean.

[12]. According to a study conducted by BRRI, the yield gap in rice was about $1.74 \mathrm{t} \cdot \mathrm{ha}^{-1}$ and at least Tk. 1260 billion could be earned from the additional production annually by minimizing the yield gap [13]. It is, therefore, essential to minimize the yield gaps and increase the productivity of the crops to feed the growing population of the country.

\section{Methodology}

\subsection{Sample Size and Location}

The study was conducted in eight Binadhan-10 \& Binadhan-11 growing areas in Bangladesh. In this study four districts namely Satkhira, Khulna, Barishal, and Cox's Bazar were used for Binadhan-10 and Mymensingh, Jamalpur, Sherpur and Sunamgonj were taken for Binadhan-11. It is based on primary level data from eight sub-districts among the study areas (Figure 4) and there were 240 (30 from each location) randomly selected farmers to conduct the study.

\subsection{Analytical Technique}

In the study, the concept of yield gap as suggested by Zandstra et al. (1981) [14] was used. Total yield gap can be decomposed into two parts i.e. Yield gap I and Yield gap II. Yield Gap I refer to the difference between research station's yield 


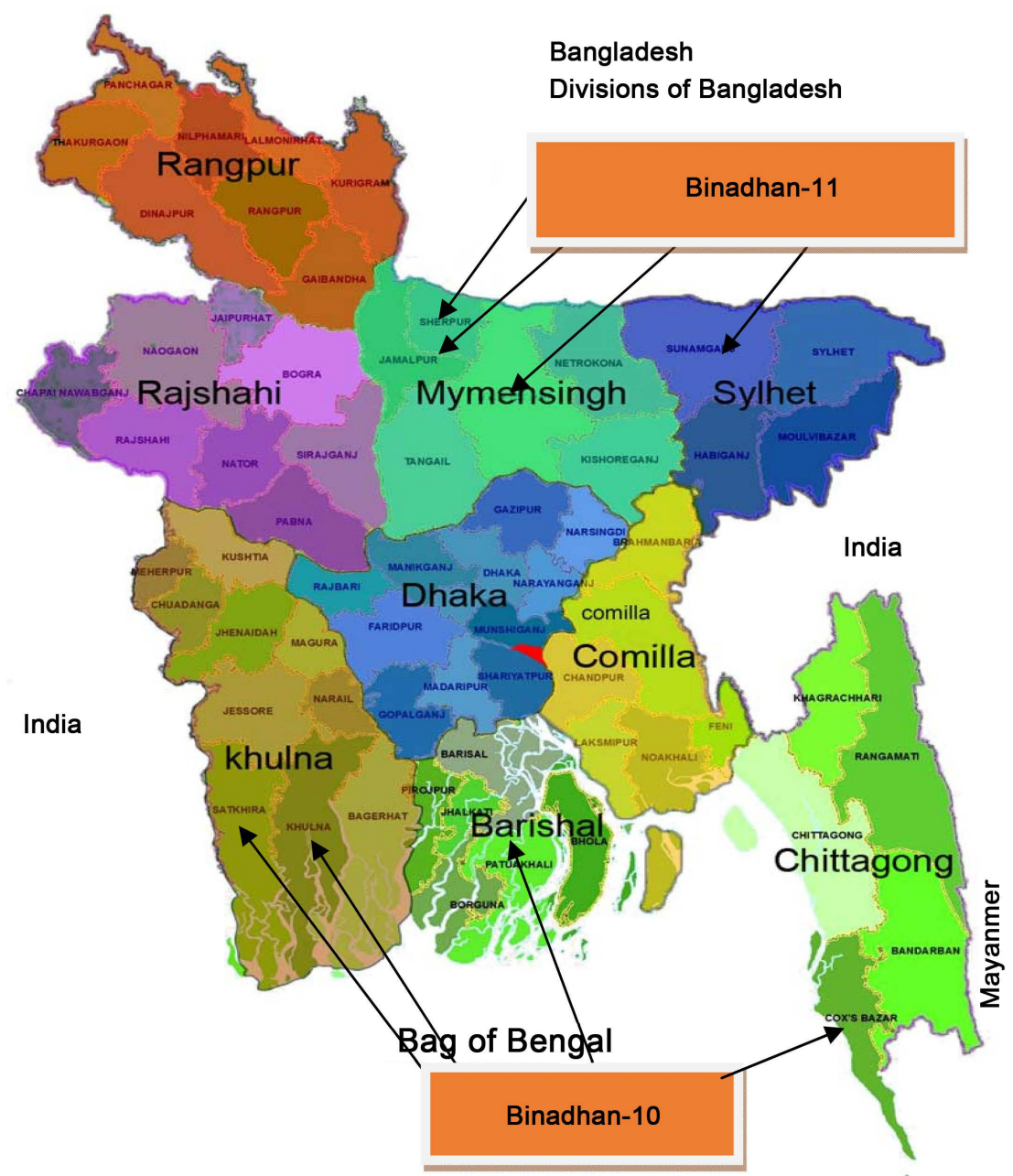

Figure 4. Different Study areas of stress tolerant rice varieties in Bangladesh. Source: www.banglapedia+map,online_chips:scientific+diagram.

and potential farm yield obtained at demonstration plots, while Yield Gap II, reflecting the effects of biophysical and socio-economic constraints, was the difference between yield obtained at the nearest demonstration plot and actual yield obtained on farmers' fields. The yield gaps were estimated as follows:

$$
\begin{gathered}
\text { Yield Gap I }=\left[\left(Y_{R}-Y_{D}\right) / Y_{R}\right] \times 100 \\
\text { Yield Gap II }=\left[\left(Y_{D}-Y_{F}\right) / Y_{D}\right] \times 100
\end{gathered}
$$

where,

$Y_{R}$ is the research station yield,

$Y_{D}$ is the demonstration plot yield, and

$Y_{F}$ is the actual farm yield.

\section{Results and Discussion}

The results presented in Table 2 showed that the highest yield was obtained in Barishal $\left(5.98 \mathrm{t} \cdot \mathrm{ha}^{-1}\right)$ followed by Khulna $\left(5.23 \mathrm{t} \cdot \mathrm{ha}^{-1}\right)$, Satkhira $\left(4.45 \mathrm{t} \cdot \mathrm{ha}^{-1}\right)$, 
Table 2. Estimated yield gap of Binadhan-10 in different locations.

\begin{tabular}{|c|c|c|c|c|c|}
\hline Particular & Barishal & Khulna & Satkhira & Cox’s Bazar & Average \\
\hline Average research station yield $\left(\mathrm{Y}_{\mathrm{R}}\right)$, t.ha ${ }^{-1}$ & 7.50 & $6.25^{*}$ & 6.25 & 6.50 & 6.63 \\
\hline Average demonstration yield $\left(\mathrm{Y}_{\mathrm{D}}\right), \mathrm{t} \cdot \mathrm{ha}^{-1}$ & 7.00 & 6.0 & 5.15 & 5.61 & 5.94 \\
\hline Average actual farm yield $\left(\mathrm{Y}_{\mathrm{F}}\right), \mathrm{t} \cdot \mathrm{ha}^{-1}$ & 5.98 & 5.23 & 4.45 & 4.16 & 4.96 \\
\hline Yield gap I (\%) & $\begin{array}{c}0.50 \\
(6.66)\end{array}$ & $\begin{array}{c}0.25 \\
(4.00)\end{array}$ & $\begin{array}{c}1.10 \\
(17.6)\end{array}$ & $\begin{array}{c}0.89 \\
(13.59)\end{array}$ & $\begin{array}{c}0.69 \\
(10.33)\end{array}$ \\
\hline Yield gap II (\%) & $\begin{array}{c}1.02 \\
(14.57)\end{array}$ & $\begin{array}{c}0.77 \\
(12.83)\end{array}$ & $\begin{array}{c}0.70 \\
(13.59)\end{array}$ & $\begin{array}{c}1.45 \\
(25.84)\end{array}$ & $\begin{array}{c}0.99 \\
(16.58)\end{array}$ \\
\hline Total yield gap (\%) & $\begin{array}{c}1.52 \\
(21.23)\end{array}$ & $\begin{array}{c}1.02 \\
(16.83)\end{array}$ & $\begin{array}{c}1.80 \\
(31.19)\end{array}$ & $\begin{array}{c}2.34 \\
(39.53)\end{array}$ & $\begin{array}{c}1.67 \\
(26.92)\end{array}$ \\
\hline
\end{tabular}

*Indicates the value of nearest sub-stations; Source: Author's calculation based on field survey, September 2018 .

Cox's Bazar (4.16 thha $\left.{ }^{-1}\right)$ district. The average yield of Binadhan-10 was 4.96 $\mathrm{t} \cdot \mathrm{ha}^{-1}$.

In Figure 5, the green, red \& blue area of diagram indicating actual farm yield, demonstration yield and research station yield, respectively and in Figure 6 the green, red \& blue area of diagram indicating total yield gap, yield gap II and yield gap I, respectively in different districts of Bindhan-10 in Bangladesh.

As seen from Figure 6, the estimated yield gap I was $0.69 \mathrm{t} \cdot \mathrm{ha}^{-1}(10.33 \%$.) and yield gap II was $0.99 \mathrm{t} \cdot \mathrm{ha}^{-1}\left(16.58 \%\right.$.). The lowest gap was $1.02 \mathrm{t} \cdot \mathrm{ha}^{-1}(16.83 \%)$ found in Khulna district and it was the highest $2.34 \mathrm{t}^{-\mathrm{ha}^{-1}}$ (39.53\%) in case of Cox's Bazar district. Considering all, the average yield gap was $1.67 \mathrm{t} \cdot \mathrm{ha}^{-1}$ (26.92\%) and much scope for yield enhancement in the variety.

The results presented in Table 3 showed that the highest yield was obtained from Sherpur (4.08 th.ha $\left.{ }^{-1}\right)$ followed by Sunamgonj (4.03 tha $\left.{ }^{-1}\right)$, Jamalpur (3.99 $\left.\mathrm{t} \cdot \mathrm{ha}^{-1}\right)$ and Mymensingh (3.87 tha $\left.{ }^{-1}\right)$ district. The average yield of Binadhan-11 was $4.00 \mathrm{t} \cdot \mathrm{ha}^{-1}$.

In Figure 7, the green, red \& blue area of diagram indicating actual farm yield, demonstration yield and research station yield, respectively and in Figure 8 the green, red \& blue area of diagram indicating total yield gap, yield gap II and yield gap I, respectively in different districts of Binadhan-11 in Bangladesh.

As seen from Figure 8 the estimated yield gap I was $0.69 \mathrm{t} \cdot \mathrm{ha}^{-1}(12.57 \%)$ and yield gap II was $0.83 \mathrm{t} \cdot \mathrm{ha}^{-1}(17.18 \%)$. The lowest gap was $1.42 \mathrm{t} \cdot \mathrm{ha}^{-1}(27.74 \%)$ observed in Sherpur district and it was the highest $1.73 \mathrm{t} \cdot \mathrm{ha}^{-1}(33.64 \%)$ in case of Mymensingh district. Considering all, the average yield gap was $1.52 \mathrm{t} \cdot \mathrm{ha}^{-1}$ (29.76\%) and much scope for yield enhancement in the variety.

Major factors that were responsible for the yield gap of Binadhan-10 were described in Table 4. It was found from the Table 4, most of the farmers did not consider the recommended doses of seed rate, fertilizer and seedling age. The average seed rate was $40.60 \mathrm{Kg} \cdot \mathrm{ha}^{-1}$, Urea $162.07 \mathrm{Kg} \cdot \mathrm{ha}^{-1}$, TSP $99.62 \mathrm{Kg} \cdot \mathrm{ha}^{-1}$, MoP $62.07 \mathrm{Kg} \cdot \mathrm{ha}^{-1}$ and seedling age 29.45 days respectively, indicates that they are either below or above the recommendation. 


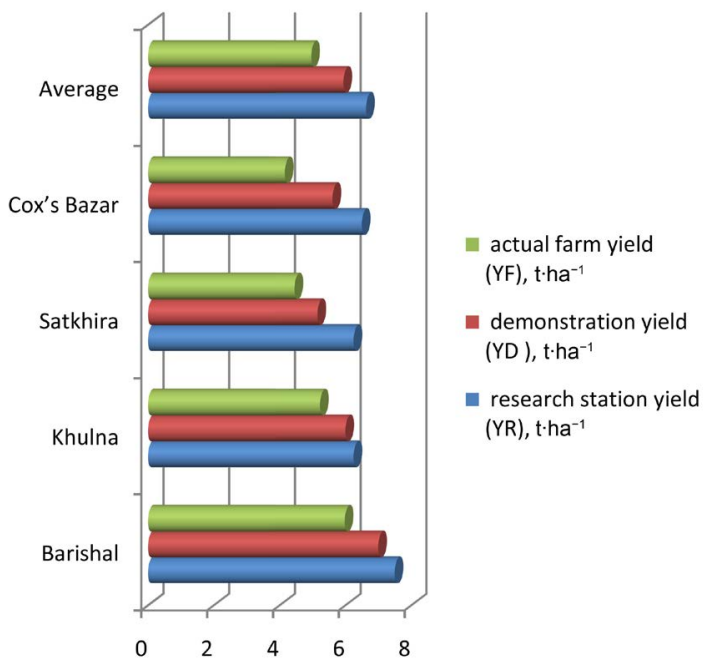

Figure 5. Yield of Binadhan-10 in different districts of Bangladesh.

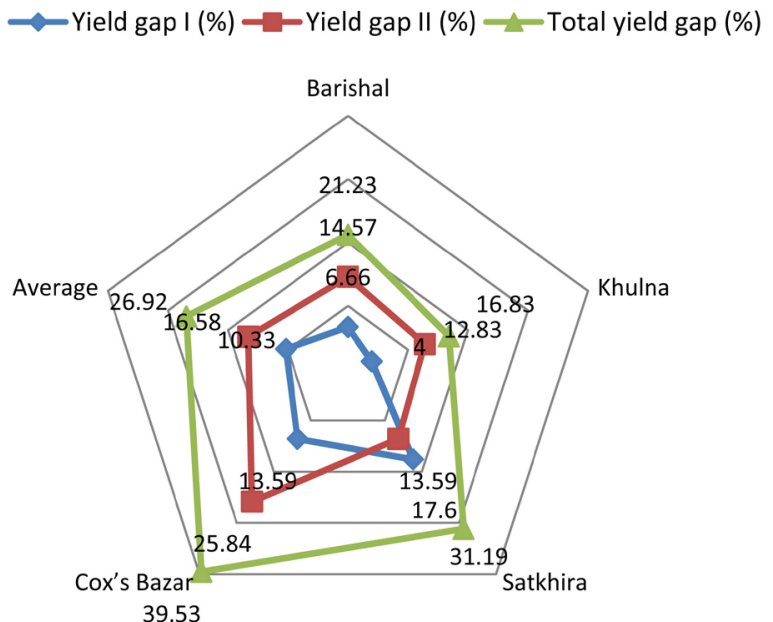

Figure 6. Yield gap of Binadhan-10 indifferent districts of Bangladesh.

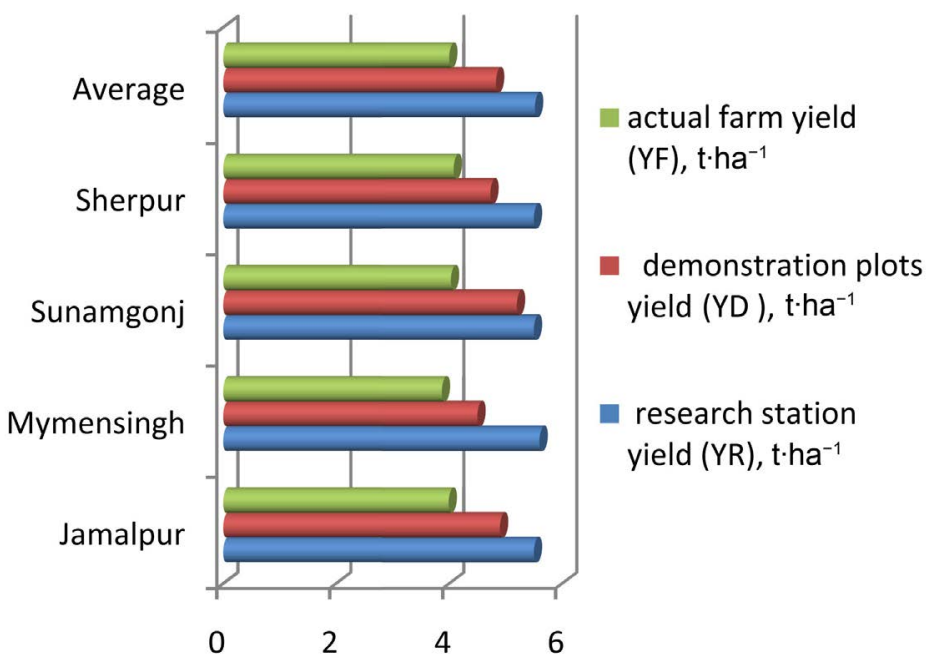

Figure 7. Yield of Binadhan-11 in different districts of Bangladesh. 


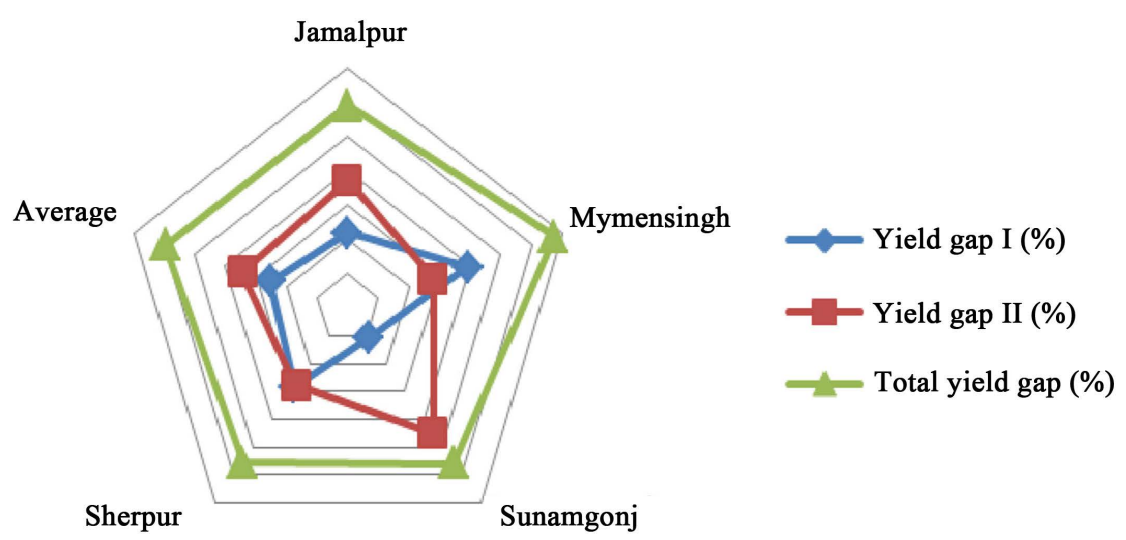

Figure 8. Yield gap of Binadhan-11 indifferent districts of Bangladesh.

Table 3. Estimated yield gap of Binadhan-11 in different locations.

\begin{tabular}{|c|c|c|c|c|c|}
\hline Particular & Jamalpur & Mymensingh & Sunamgonj & Sherpur & Average \\
\hline $\begin{array}{c}\text { Average yield of } \\
\text { research station }\left(Y_{R}\right), t \cdot h a^{-1}\end{array}$ & 5.5 & 5.60 & 5.50 & 5.50 & 5.52 \\
\hline $\begin{array}{l}\text { Average yield of demonstration } \\
\text { plots }\left(\mathrm{Y}_{\mathrm{D}}\right), \mathrm{t} \cdot \mathrm{ha}^{-1}\end{array}$ & 4.90 & 4.50 & 5.20 & 4.73 & 4.83 \\
\hline $\begin{array}{c}\text { Average yield of } \\
\text { actual farm }\left(\mathrm{Y}_{\mathrm{F}}\right), \mathrm{t} \cdot \mathrm{ha}^{-1}\end{array}$ & 3.99 & 3.87 & 4.03 & 4.08 & 4.00 \\
\hline Yield gap I (\%) & $\begin{array}{c}0.6 \\
(10.90)\end{array}$ & $\begin{array}{c}1.1 \\
(19.64)\end{array}$ & $\begin{array}{c}0.30 \\
(5.45)\end{array}$ & $\begin{array}{l}0.77 \\
(14)\end{array}$ & $\begin{array}{c}0.69 \\
(12.57)\end{array}$ \\
\hline Yield gap II (\%) & $\begin{array}{c}0.91 \\
(18.57)\end{array}$ & $\begin{array}{l}0.63 \\
(14)\end{array}$ & $\begin{array}{c}1.17 \\
(22.50)\end{array}$ & $\begin{array}{c}0.65 \\
(13.74)\end{array}$ & $\begin{array}{c}0.83 \\
(17.18)\end{array}$ \\
\hline Total yield gap (\%) & $\begin{array}{c}1.51 \\
(29.48)\end{array}$ & $\begin{array}{c}1.73 \\
(33.64)\end{array}$ & $\begin{array}{c}1.44 \\
(27.95)\end{array}$ & $\begin{array}{l}(1.42) \\
27.74\end{array}$ & $\begin{array}{c}1.52 \\
(29.76)\end{array}$ \\
\hline
\end{tabular}

Source: Author's calculation based on field survey, September 2018.

Table 4. Input-use pattern of Binadhan-10 growing farmers.

\begin{tabular}{cccccc}
\hline Factors & $\begin{array}{c}\text { Seed } \\
\mathrm{Kg}^{\prime} \mathrm{ha}^{-1}\end{array}$ & $\begin{array}{c}\text { Urea } \\
\mathrm{Kg}^{\prime} \mathrm{ha}^{-1}\end{array}$ & $\begin{array}{c}\text { MoP } \\
\mathrm{Kg}^{-} \mathrm{ha}^{-1}\end{array}$ & $\begin{array}{c}\text { Tsp } \\
\mathrm{Kg}^{-h^{-1}}\end{array}$ & $\begin{array}{c}\text { Seedling age } \\
(\text { days })\end{array}$ \\
\hline Recommended & $25-30$ & $210-220$ & $85-100$ & $110-115$ & $30-35$ \\
Khulna & 42 & 172.12 & 56.64 & 91.21 & 29.33 \\
Sathkhira & 38 & 170.619 & 82.48 & 121.8703 & 29.12 \\
Barishal & 40.23 & 171.66 & 53.46 & 102.76 & 31.05 \\
Cox's Bazar & 42.16 & 133.9 & 55.71 & 82.64 & 28.30 \\
Average & 40.60 & 162.02 & 62.07 & 99.62 & 29.45 \\
\hline
\end{tabular}

Source: Author's calculation based on field survey, September 2018.

In case of Binadhan-11 average seed rate was $42.54 \mathrm{Kg} \cdot \mathrm{ha}^{-1}$, Urea 126.43 $\mathrm{Kg} \cdot \mathrm{ha}^{-1}$, Tsp $72.40 \mathrm{Kg} \cdot \mathrm{ha}^{-1}$, MoP $56.80 \mathrm{Kg} \cdot \mathrm{ha}^{-1}$ and seedling age 28.27 days respectively, indicating except seedling age, they are either below or above the recommendation (Table 5). 
Table 5. Input-use pattern of Binadhan-11 growing farmers.

\begin{tabular}{|c|c|c|c|c|c|}
\hline Factors & $\begin{array}{c}\text { Seed } \\
\mathrm{Kg} \cdot \mathrm{ha}^{-1}\end{array}$ & 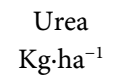 & $\begin{array}{c}\mathrm{MoP} \\
\mathrm{Kg} \cdot \mathrm{ha}^{-1}\end{array}$ & $\begin{array}{c}\text { Tsp } \\
\mathrm{Kg} \cdot \mathrm{ha}^{-1}\end{array}$ & $\begin{array}{l}\text { seedling age } \\
\text { (days) }\end{array}$ \\
\hline Recommended & $25-30$ & $150-180$ & $50-70$ & $110-120$ & $25-30$ \\
\hline Jamalpur & 40.37 & 169.28 & 58.60 & 76.80 & 27.73 \\
\hline Mymensingh & 44.04 & 144.04 & 59.79 & 64.69 & 28.46 \\
\hline Sunamgonj & 42.45 & 89.88 & 38.43 & 61.57 & 28.16 \\
\hline Sherpur & 43.31 & 102.52 & 70.38 & 86.57 & 28.73 \\
\hline Average & 42.54 & 126.43 & 56.80 & 72.40 & 28.27 \\
\hline
\end{tabular}

Source: Author's calculation based on field survey, September 2018.

The responded were classified as affected and not affected by salinity in case of Binadhan-10 producing farmers and not affected, affected and highly affected by flood in case of Binadhan-11 producing farmers to identify the factors responsible for yield gap of the varieties. In case of non-saline areas $26.67 \%$ farmer's average yield was $6.3 \mathrm{t} \cdot \mathrm{ha}^{-1}$ by using $41.62 \mathrm{Kg} \cdot \mathrm{ha}^{-1}$ seed rate which was greater than the recommended dose and other major inputs such as Urea $187.46 \mathrm{Kg} \cdot \mathrm{ha}^{-1}$, TSP. $103 \mathrm{Kg} \cdot \mathrm{ha}^{-1}$, Mop $53.49 \mathrm{Kg} \cdot \mathrm{ha}^{-1}$, Gypsum $22.74 \mathrm{Kg} \cdot \mathrm{ha}^{-1}$ were also below the required level.

The seedling age of the Binadhan-10 was 32.03 days was in the recommended range, land cultivated by power tiller, weeding, pesticide and insecticide spray were $3.23,1.57,0.38$ and 1.12 times, respectively. The average farmers yield gap

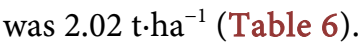

In case of saline areas farmer's average yield was $4.7 \mathrm{t} \cdot \mathrm{ha}^{-1}$ and using 39.59 $\mathrm{Kg} \cdot \mathrm{ha}^{-1}$ seed rate which was greater than the recommended dose and other major inputs for Binadhan-10 cultivation such as Urea $155.56 \mathrm{Kg} \cdot \mathrm{ha}^{-1}$, TSP 99.47 $\mathrm{Kg} \cdot \mathrm{ha}^{-1}$, Mop $69.24 \mathrm{Kg} \cdot \mathrm{ha}^{-1}$, Gypsum $27.85 \mathrm{Kg} \cdot \mathrm{ha}^{-1}$ were also below the required level. The seedling age of the Binadhan-10 was 27.90 days, land cultivated by power tiller, weeding, pesticide and insecticide spray were 3.36, 1.74, 0.16 and 2.16 times, respectively. The average farmers yield gap was $1.2 \mathrm{t} \cdot \mathrm{ha}^{-1}$. It can be seen that soil fertility was important factor for both of the variety. The overall soil fertility was the highest in case of not affected farmer as well as their yield was also the highest $6.32 \mathrm{t} \cdot \mathrm{ha}^{-1}$.

In case of submergence tolerant rice variety and not affected by flood, $16.66 \%$ Binadhan-11 producing farmer getting yield $4.1 \mathrm{t} \cdot \mathrm{ha}^{-1}$ by using $40.30 \mathrm{Kg} \cdot \mathrm{ha}^{-1}$ seed rate which was higher than the recommended dose and other major inputs such as Urea 107.83 $\mathrm{Kg} \cdot \mathrm{ha}^{-1}$, TSP $86.49 \mathrm{Kg} \cdot \mathrm{ha}^{-1}$, MoP $81.50 \mathrm{Kg} \cdot \mathrm{ha}^{-1}$, Gypsum $11.51 \mathrm{Kg} \cdot \mathrm{ha}^{-1}$ were either below or above the required level. The seedling age of the Binadhan-11 was 28.72 days, land cultivated by power tiller, weeding, pesticide and insecticide spray were $3.63,1.63,0.67$ and 1.11 times, respectively,. The average farmers yield gap was $1.42 \mathrm{t} \cdot \mathrm{ha}^{-1}$ (Table 7$)$. 
Table 6. Factors responsible for yield gap of Binadhan-10 cultivation in the study areas.

\begin{tabular}{|c|c|c|c|c|}
\hline Item & recommended & $\begin{array}{c}\text { Farmer } \\
(\text { no) }(\%)\end{array}$ & Mean & $\begin{array}{l}\text { Standard } \\
\text { deviation }\end{array}$ \\
\hline 1. Yield $\left(\mathrm{Kg} \cdot \mathrm{ha}^{-1}\right)$ & 7500 & & 6322.41 & 2319.50 \\
\hline 1.1. Yield gap & - & & 2023.69 & 1605.65 \\
\hline 2. Seed rate $\left(\mathrm{Kg} \cdot \mathrm{ha}^{-1}\right)$ & $25-30$ & & 41.62 & 17.21 \\
\hline 3. Power tiller (no.) & - & & 3.23 & 0.82 \\
\hline \multicolumn{5}{|l|}{ 4. Fertilizer $\left(\mathrm{Kg} \cdot \mathrm{ha}^{-1}\right)$} \\
\hline & & Not affected & 187.46 & 260.91 \\
\hline - $\quad$ TSP & $110-115$ & (32) (26.67\%) & 103.48 & 69.56 \\
\hline - $\mathrm{MoP}$ & $85-100$ & & 53.49 & 52.18 \\
\hline - Gypsum & $50-60$ & & 22.74 & 30.13 \\
\hline 5. Seedling age (days) & $30-35$ & & 32.03 & 5.84 \\
\hline 6. Weeding (no.) & - & & 1.57 & 0.89 \\
\hline 7. Pesticide & - & & 0.38 & 0.90 \\
\hline 8. Insecticide & - & & 1.12 & 1.16 \\
\hline 9. Soil fertility & & & 1.90 & 0.40 \\
\hline Item & recommended & Farmer (no) (\%) & Mean & $\begin{array}{l}\text { Standard } \\
\text { deviation }\end{array}$ \\
\hline 1. Yield $\left(\mathrm{Kg} \cdot \mathrm{ha}^{-1}\right)$ & 5500 & & 4692.58 & 1225.66 \\
\hline 1.1. Yield gap & - & & 1191.39 & 852.01 \\
\hline 2. Seed rate $\left(\mathrm{Kg} \cdot \mathrm{ha}^{-1}\right)$ & $25-30$ & & 39.59 & 23.48 \\
\hline 3. Power tiller (no.) & - & & 3.36 & 0.76 \\
\hline \multicolumn{5}{|l|}{ 4. Fertilizer $\left(\mathrm{Kg} \cdot \mathrm{ha}^{-1}\right)$} \\
\hline - Urea & $210-220$ & & 155.56 & 55.04 \\
\hline - $\quad$ TSP & $110-115$ & $\begin{array}{c}\text { Affected } \\
\text { (88) }(73.33 \%)\end{array}$ & 99.47 & 39.99 \\
\hline - $\mathrm{MoP}$ & $85-100$ & (88) (73.33\%) & 69.24 & 35.74 \\
\hline - Gypsum & $50-60$ & & 27.85 & 49.92 \\
\hline 5. Seedling age (days) & $30-35$ & & 27.90 & 3.52 \\
\hline 6. Weeding (no.) & - & & 1.74 & 0.73 \\
\hline 7. Pesticide & - & & 0.16 & 0.58 \\
\hline 8. Insecticide & - & & 2.16 & 0.92 \\
\hline 9. Soil fertility & & & 1.65 & 0.75 \\
\hline
\end{tabular}

Source: Author's calculation based on field survey, September 2018.

In case of medium flood affected area (affected 1 - 10 days) $68.33 \%$ Binadhan-11 farmer also getting yield $4.1 \mathrm{t} \cdot \mathrm{ha}^{-1}$ using $45.00 \mathrm{Kg} \cdot \mathrm{ha}^{-1}$ seed rate which was higher than the recommended dose and other major inputs such as Urea 142.23 Kg.ha ${ }^{-1}$, Tsp $80.59 \mathrm{Kg} \cdot \mathrm{ha}^{-1}$, Mop $57.40 \mathrm{Kg} \cdot \mathrm{ha}^{-1}$, Gypsum $4.87 \mathrm{Kg} \cdot \mathrm{ha}^{-1}$ were also below the required level except mop. The seedling age of the Binadhan-11 was 28.04 days, land cultivated by power tiller, weeding, pesticide and insecticide 
Table 7. Factors responsible for yield gap of Binadhan-11 cultivation in the study areas.

\begin{tabular}{|c|c|c|c|c|}
\hline Gap in practices & Recommended & $\begin{array}{l}\text { Farmer } \\
\text { (no) (\%) }\end{array}$ & Mean & $\begin{array}{l}\text { Standard } \\
\text { deviation }\end{array}$ \\
\hline 1. Yield $\left(\mathrm{Kg} \cdot \mathrm{ha}^{-1}\right)$ & 5500 & & 4075.82 & 809.44 \\
\hline 1.1. Yield gap & - & & 1427.17 & 809.44 \\
\hline 2. Seed rate $\left(\mathrm{Kg} \cdot \mathrm{ha}^{-1}\right)$ & $25-30$ & & 40.30 & 10.87 \\
\hline 3. Power tiller (no.) & - & & 3.63 & 0.49 \\
\hline \multicolumn{5}{|l|}{ 4. Fertilizer $\left(\mathrm{Kg} \cdot \mathrm{ha}^{-1}\right)$} \\
\hline - Urea & $150-180$ & $\begin{array}{l}\text { Not affected } \\
(20)(16.66 \%)\end{array}$ & 107.83 & 54.47 \\
\hline - $\mathrm{TSP}$ & $110-120$ & & 86.49 & 49.09 \\
\hline - $\mathrm{MoP}$ & $50-70$ & & 81.50 & 50.22 \\
\hline - Gypsum & $20-30$ & & 11.51 & 27.68 \\
\hline 5. Seedling age (days) & $25-30$ & & 28.72 & 2.29 \\
\hline 6. Weeding (no.) & - & & 1.63 & 0.49 \\
\hline 7. Pesticide & - & & 0.67 & 0.84 \\
\hline 8. Insecticide & - & & 1.11 & 0.82 \\
\hline 9. Soil fertility & - & & 1.63 & 0.59 \\
\hline Gap in Practices & recommended & Farmer (no) (\%) & Mean & $\begin{array}{l}\text { Standard } \\
\text { deviation }\end{array}$ \\
\hline 1. Yield $\left(\mathrm{Kg} \cdot \mathrm{ha}^{-1}\right)$ & 4500 & & 4111.89 & 1094.36 \\
\hline 1.1. Yield gap & - & & 388.10 & 1094.36 \\
\hline 2. Seed rate $\left(\mathrm{Kg} \cdot \mathrm{ha}^{-1}\right)$ & $25-30$ & & 45.00 & 16.45 \\
\hline 3. Power tiller (no.) & - & & 3.33 & 0.88 \\
\hline \multicolumn{5}{|l|}{ 4. Fertilizer $\left(\mathrm{Kg} \cdot \mathrm{ha}^{-1}\right)$} \\
\hline - Urea & $150-180$ & $\begin{array}{c}\text { Affected } \\
(1-10 \text { days })\end{array}$ & 142.23 & 70.20 \\
\hline - $\quad$ TSP & $110-120$ & (82) $(68.33 \%)$ & 80.59 & 45.31 \\
\hline • $\mathrm{MoP}$ & $50-70$ & & 57.40 & 37.93 \\
\hline • Gypsum & $20-30$ & & 4.87 & 13.96 \\
\hline 5. Seedling age (days) & $25-30$ & & 28.04 & 2.59 \\
\hline 6. Weeding (no.) & - & & 1.58 & 0.66 \\
\hline 7. Pesticide & - & & 0.24 & 0.64 \\
\hline 8. Insecticide & - & & 1.04 & 1.01 \\
\hline 9. Soil fertility & - & & 1.85 & 0.38 \\
\hline Gap in Practices & recommended & Farmer (no) (\%) & Mean & $\begin{array}{l}\text { Standard } \\
\text { deviation }\end{array}$ \\
\hline 1. Yield $\left(\mathrm{Kg} \cdot \mathrm{ha}^{-1}\right)$ & 4500 & & 3690.63 & 934.55 \\
\hline 1.1. Yield gap & - & Highly affected & 809.36 & 934.55 \\
\hline 2. Seed rate $\left(\mathrm{Kg} \cdot \mathrm{ha}^{-1}\right)$ & $25-30$ & (11 - 20 days $)$ & 42.32 & 15.22 \\
\hline 3. Power tiller (no.) & - & (18) (15\%) & 3.05 & 0.80 \\
\hline 4. Fertilizer $\left(\mathrm{Kg} \cdot \mathrm{ha}^{-1}\right)$ & & & & \\
\hline
\end{tabular}




\begin{tabular}{lccc} 
Continued & & & \\
\hline - Urea & $150-180$ & 104.30 & 52.38 \\
- TSP & $110-120$ & 57.57 & 44.69 \\
- MoP & $50-70$ & 48.70 & 48.75 \\
- Gypsum & $20-30$ & 18.03 & 28.60 \\
5. Seedling age (days) & $25-30$ & 27.33 & 2.54 \\
6. Weeding (no.) & - & 1.16 & 0.78 \\
7. Pesticide & - & 0.61 & 0.84 \\
8. Insecticide & - & 0.50 & 0.78 \\
9. Soil fertility & - & 1.22 & 0.73
\end{tabular}

Source: Author's calculation based on field survey, September 2018.

spray were $3.33,1.58,0.24$ and 1.04 times, respectively. The average farmers yield gap was $0.38 \mathrm{t} \cdot \mathrm{ha}^{-1}$.

In case of highly flood affected area (affected 11 - 20 days) 15\% Binadhan-11 producing farmer getting yield $3.7 \mathrm{t} \cdot \mathrm{ha}^{-1}$ by using $42.32 \mathrm{Kg} \cdot \mathrm{ha}^{-1}$ seed rate which was higher than the recommended dose and other major inputs such as Urea 104.30 Kg.ha ${ }^{-1}$, TSP $57.57 \mathrm{Kg} \cdot \mathrm{ha}^{-1}$, MoP $48.70 \mathrm{Kg} \cdot \mathrm{ha}^{-1}$, Gypsum $18.03 \mathrm{Kg} \cdot \mathrm{ha}^{-1}$ were below the required level. The seedling age of the Binadhan-11 was 27.33 days, land cultivated by power tiller, weeding, pesticide and insecticide spray were $3.05,1.16,0.61$ and 0.50 times, respectively. The average farmers yield gap was $0.80 \mathrm{t} \cdot \mathrm{ha}^{-1}$.

It can be seen that soil fertility was important factor for both of the variety. The overall soil fertility was the highest in case of medium flood affected farmer as well as their yield was also the highest $4.1 \mathrm{t} \cdot \mathrm{ha}^{-1}$.

Correlation between different factors in rice cultivation and yield in the study areas

Correlation co-efficient were computed to ascertain the relationships between different factors in rice cultivation and yield in these varieties. The findings presented in Table 8 show that all the inputs in case of Binadhan-10 cultivation had significant positive relationships with the yield. This meant that yield of rice increased with the increase level of inputs.

The results showed that all the inputs in Binadhan-11 cultivation had significant positive relationships with the yield (Table 9). This meant that yield of rice increased with the increase level of inputs.

\section{Some policy guidelines to reduce the Yield Gap}

At fast, Farmers should follow the recommended doses of inputs in the production process. Besides quality seed must be ensured in timely and awareness, motivation as well as extension programme also be increased. Secondly some farmers noticed about high price of labour in sowing and harvesting time, reluctant in adopting new technology and adulterated fertilizer \& insecticide also responsible for achieving targeted yield. Thus it is necessary to provide adequate 
Table 8. Correlation between yield and factors used by the farmers among the study areas for Binadhan-10.

\begin{tabular}{ccc}
\hline Sl. No. & Factors & Correlation co-efficient $(\mathbf{r})$ \\
\hline 1. & Seed rate & $0.606^{* * *}$ \\
2. & Power tiller & $0.210^{* *}$ \\
3. & Urea & $0.550^{* * *}$ \\
4. & TSP & $0.551^{* * *}$ \\
5. & MoP & $0.420^{* *}$ \\
6. & Zypsum & $0.251^{* *}$ \\
7. & Quantity of irrigation & $0.168^{*}$ \\
8. & farm size & $0.727^{* * *}$
\end{tabular}

Note: “*”, “**” and “***»" indicate significant at 10\%, 5\% and 1\% level. Source: Author's calculation based on field survey, September 2018.

Table 9. Correlation between yield and factors used by the farmers among the study areas for Binadhan-11.

\begin{tabular}{ccc}
\hline Sl No. & Factors & Correlation co-efficient $(\mathbf{r})$ \\
\hline 1. & Seed rate & $0.739^{* * *}$ \\
2. & Power tiller & $0.184^{* *}$ \\
3. & Urea & $0.674^{* * *}$ \\
4. & TSP & $0.578^{* * *}$ \\
5. & MoP & $0.484^{* * *}$ \\
6. & Zypsum & $0.289^{* *}$ \\
7. & farm size & $0.912^{* * *}$
\end{tabular}

Note: “**” and “***” indicate significant at $5 \%$ and $1 \%$ level. Source: Author's calculation based on field survey, September 2018.

labour facilities during sowing and harvesting time and government should take appropriate action through law enforcement team to stop the use of adulterated fertilizer and insecticide throughout the country.

\section{Conclusion}

Increasing yield as well as agricultural productivity is urgent for economic growth and development for any country in the world. The study found that in Bangladesh, we are losing $1.67 \mathrm{t} \cdot \mathrm{ha}^{-1}$ (26.92\%) yield of Binadhan-10 in Boro season and $1.52 \mathrm{t} \cdot \mathrm{ha}^{-1}$ (29.76\%) of Binadhan-11 in Aman season. If we could reduce these gaps, our total production per year will be increased by $3.19 \mathrm{t} \cdot \mathrm{ha}^{-1}$ which will support in achieving food security as well as Sustainable Development Goals (SDGs).

\section{Highlights}

The study revealed that in case of submergence tolerant rice variety Binadhan-11, per hectare yield gap I was 0.69 tonne (12.57\%) and yield gap II was 
0.83 tonne $(17.18 \%)$. The lowest gap was $1.42 \mathrm{t} \cdot \mathrm{ha}^{-1}(27.74 \%)$ observed in Sherpur district and it was the highest $1.73 \mathrm{t} \cdot \mathrm{ha}^{-1}$ (33.64\%) in case of Mymensingh district. Considering all, the average yield gap for Binadhan-11 was $1.52 \mathrm{t} \cdot \mathrm{ha}^{-1}$ i.e. $29.76 \%$ in Aman season.

For Binadhan-10, a salt tolerant rice variety the estimated yield gap I was 0.69 tonne per hectare $(10.33 \%)$ and yield gap II was $0.99 \mathrm{t} \cdot \mathrm{ha}^{-1}(16.58 \%)$ for $\mathrm{Bi}$ nadhan-10. The lowest gap was $1.02 \mathrm{t} \cdot \mathrm{ha}^{-1}(16.83 \%)$ found in Khulna district and it was the highest $2.34 \mathrm{t} \cdot \mathrm{ha}^{-1}(39.53 \%)$ in case of Cox's Bazar district. Considering all, the average yield gap was $1.67 \mathrm{t} \cdot \mathrm{ha}^{-1}(26.92 \%)$ in Boro season.

If we could reduce these gaps, our total production per year will be increased by $3.19 \mathrm{t} \cdot \mathrm{ha}^{-1}$ which will support in achieving food security as well as Sustainable Development Goals (SDGs).

\section{Conflicts of Interest}

The authors declare no conflicts of interest regarding the publication of this paper.

\section{References}

[1] Hossain, M. (1995) Sustaining Food Security for Fragile Environments in Asia: Achievements, Challenges, and Implications for Rice Research. Fragile Lives in Fragile Ecosystems, IRRI, Los Baños, 3-23.

[2] Swaminathan, M.S. (1998) Issues and Challenges in Sustainable Increased Rice Production and the Role of Rice in Human Nutrition in the World. International Rice Comission 98/4-2, Cairo, 23.

[3] Word Bank Population Projection, 1994-95, 20-24.

[4] https://journals.plos.org/plosone/article/figure?id=10.1371/journal.pone.0066428. g002

[5] Alam, M. (2006) Factors Affecting Yield Gap and Efficiency in Rice Productions in Some Selected Areas of Bangladesh. A Thesis Submitted for the Degree of Ph.D. Department of Economics, Jahangir Nagar University, Bangladesh.

[6] OFRD, BARI (2007-2008) Yield Gap Analysis of Mustard Production under Different Management Practices. Annual Report of OFRD, Bari, 385-387.

[7] OFRD (On-Farm Research Division) (2008-2009) Yield Gap Analysis of Mustard in the Farmers' Fields. Annual Report of OFRD, Bari, 384-386.

[8] Roy, I. (1997) Stagnating Productivity in Crop Agriculture. The Quest for Sources of Growth. Environment and Agricultural Productivity in Bangladesh. Bangladesh Academy of Agriculture (BAAG), 25-48.

[9] Matin, M.A., Anwarul Huq, A.S.M., Hussain, M.S., Karim, Md.R. and Baksh, E. (1996) Farm Level Yield Analysis of Tomato Cultivation in Selected Areas of Bangladesh: An Economic Profile. Bangladesh Journal of Agricultural Research, 21, 110-117.

[10] Mondal, M.H. (2011) Causes of Yield Gaps and Strategies for Minimizing the Gaps in Different Crops of Bangladesh. Bangladesh Journal of Agricultural Research, 36, 469-476. https://doi.org/10.3329/bjar.v36i3.9274 
[11] Mishra, D.K., Tailor, R.S., Pathak, G. and Deshwal, A. (2007) Yield Gap Analysis of Blight Disease Management in Potato through Front Line Demonstration. Indian Research Journal of Extension Education, 7, 82-84.

[12] Agarwal, P.K., Hebbar, K.B., Venugopalan, M.V., Rani, S., Bala, A., Biswal, A. and Wani, S.P. (2008) Quantification of Yield Gaps in Rainfed Rice, Wheat, Cotton and Mustard in India. ICRISAT Global Theme on Agro-Ecosystems Report\# 43.

[13] BRRI (2011) Study Findings Presented in a Workshop Held in May 2011 on "Minimizing Rice Yield Gap" at BRRI, Gazipur.

[14] Zandstra, H.G., Price, E.C., Litsinger, J.A. and Morris, R.A. (1981) A Methodology for On-Farm Cropping Systems Research. IRRI, Manila, 147 p. 